\title{
The Use of Abbreviations in English-Medium Astrophysics Research Paper Titles: A Problematic Issue
}

\author{
David I. Méndez (Corresponding author) \\ Department of Physics, System Engineering and Signal Theory, Polytechnic University College, University of Alicante, Spain \\ E-mail: david.mendez@ua.es \\ M. Ángeles Alcaraz \\ Department of English Studies, Faculty of Arts, University of Alicante, Spain \\ E-mail: ariza@ua.es
}

Doi:10.7575/aiac.alls.v.6n.3p.185

URL: http://dx.doi.org/10.7575/aiac.alls.v.6n.3p.185
Received: $15 / 02 / 2014$

Accepted: 21/04/2015

\begin{abstract}
In this study, we carry out a qualitative and quantitative analysis of abbreviations in 300 randomly collected research paper titles published in the most prestigious European and US-based Astrophysics journals written in English. Our main results show that the process of shortening words and groups of words is one of the most characteristic and recurrent features in Astrophysics research paper titling construction. In spite of the convenience of abbreviations as a mechanism for word-formation, some of them may pose certain difficulties of understanding and/or misinterpretation because of their specificity, ambiguity, or overlapping. To overcome these difficulties, we propose a series of options which with no doubt would lead to a better interaction among the different branches of Astrophysics in particular and of science in general and would definitely improve how research is currently performed and communicated.
\end{abstract}

Keywords: Abbreviations; Astrophysics; English; Research Papers

\section{Introduction}

In our increasingly busy world where researchers and academics are inundated by scholarly journals, efficient and effective literature searching is essential for the professional. Since Swales' (1990) seminal work on genre analysis, it has indeed been established that research papers (RPs), as the "key genre of modern knowledge creation" (Hyland, 2000 , p. 42), are the most valuable and available source of information as well as the main channel for scientists' ongoing education and for distributing new knowledge worldwide (e.g. Leventhal, 2011; Publishing Research Consortium, 2011). Nevertheless, because of the current tremendous growth in the number of periodicals published, thanks in large part to the rise of electronic publishing (Science Daily, 2008), and of the interdisciplinary nature of research, today's researchers and scientists who wish to keep up to date with new and relevant information in their field of enquiry face a truly daunting task: from the ever-increasing amount of research being published, they must indeed discriminate between what is worth reading and what is not.

Here lays the double capital role of titles in scientific research because, as Swales (1990, p. 179) so rightfully put forth, titles are both a "front and summary matter'. On the one hand, they are the gateway that represents the reader's first encounter with a document, whether it is a research article, a thesis, a conference paper, a review paper, etc. On the other hand, they allow academics decide on the relevance and usefulness of a given paper to their own area of interest. To this end, titles have to be clear, accurate and precise. As a matter of fact, the more precise and accurate titles are, the easier they will attract the attention of and inform their primary target audience, editors and reviewers, and the easier it will be for bibliographers to compile data for indexing, abstracting and other documentation purposes.

Due to their importance in academic writing, titles have been studied from a wide range of contexts and from different perspectives (cross-generic, cross-disciplinary, cross-linguistic, mono-disciplinary, etc.) ${ }^{1}$. Among the studies that have examined title wording, we can mention those by Fortanet, Coll, Palmer, and Posteguillo (1997), Fortanet, Posteguillo, Coll, and Palmer (1998), Anthony (2001), Wang and Bai (2007), or Cheng, Kuo, and Kuo (2012), to name just a few. Fortanet et al. (1997, 1998) observed that Applied Linguistics and Business and Economics titles displayed a majority of -ing forms while Chemistry and Computer Science titles showed a balanced distribution between -ing and -ed forms. Anthony (2001), who studied the length, word frequency, prepositions, and punctuation marks in the RP titles of various Computer Science sub-disciplines, discovered that there were statistically significant differences in the most common words from journal to journal, showing the sub-disciplinary content specificity. Wang and Bai (2007) also revealed that full sentence, prepositional and -ing phrase titles occurred much less frequently than compound or nominal structures. In a similar vein, Cheng, Kuo, and Kuo (2012) noted the prevalence of compound and nominal titles, as well as of -ing and prepositional phrases.

All the studies dealing with titles have shown that they vary and at the same time maintain a series of similarities across 
a number of factors and in several dimensions including functions, informativity, length, punctuation marks, structure, or wording. However, none of them has dealt with abbreviations in titles contained in RPs published in specialized journals which, on many occasions, may lead to confusion and turn these "texts in miniature" (Haggan, 2004, p. 20) into non-intelligible not only for novice, but also for expert readers. This problem is even more acute for non-English speakers when reading titles written in English. Abbreviations are all the more a problematic issue in a field such as Astrophysics, a discipline with a dual nature, i.e. a combination of Astronomy as an observational science related to the description and the classification of the universe, and of Physics, which is concerned with the basic properties of celestial objects (Pedersen, 2009). Moreover, Physics is a multidisciplinary science which combines separate branches of fields of expertise, each one with its own characteristics: Particle Physics, Relativistic Physics, Solid-state Physics, Mathematics, Chemistry, Biology, Mechanical/Electrical/Aerospace Engineering, etc.

Finally, if we consider that from a semantic and pragmatic point of view abbreviations are usually concerned with a very specialized field of application within a specific branch of research, their non-regulated use may clearly contribute to the undesirable creation of "comprehension watertight compartments" in the Astrophysics community, even among expert readers and writers. This is why we believe that Astrophysics RPs in general and Astrophysics RP titles in particular are a touchstone for a fruitful study on abbreviations so as to give, for instance, useful instructions for professional scientific writing in order to avoid the problems that may arise when not following them closely. On the other hand, it is a field which has been till now discoursively left unaddressed but for a few exceptions (Hyland, 1998; Tarone, Dwyer, Gillette \& Icke, 1998; or Méndez, Alcaraz, \& Salager-Meyer, 2014a).

Moreover, previous cross-journal and diachronic studies on three different variables, i.e. title length (Méndez, Alcaraz, \& Salager-Meyer, 2014a), authorship variations (Méndez, Alcaraz, \& Salager-Meyer, 2014b) and collaboration practices (Méndez \& Alcaraz, 2015) in Astrophysics have clearly shown that economic patterns lead the trends in astrophysical research. If we take into account that abbreviations in Astrophysics titles may be mostly related to technological progress, which in turn is definitively related to investment, we think that it is also interesting to study the distribution of abbreviations over time.

This approach would allow us to check whether the figures obtained in terms of journal comparison and evolution in the use of abbreviations would corroborate the results reached in our former studies. If it were so, our study would thus provide further insight into the structure of titles and would have both research and pedagogical implications since it may be helpful for researchers, students and teachers not only in the field of Astrophysics, but also in other disciplines.

As Astrophysics is a complex and diversified field, the present study tries to answer the following questions:

1) Are abbreviations a common feature in Astrophysics RP titles?

2) Does their use vary over time and depend on the scope of the journals analysed?

3) Does their use depend on other extra-linguistic factors?

4) May their use pose a challenge to their comprehension, even among experts in the field?

5) How may the difficulties of understanding, if any, be overcome?

\section{Corpus and methodology}

The present study adds to the previous research on the morpho-syntactic aspects of titles by examining the presence of abbreviations in a corpus of titles drawn from the four major English-medium Astrophysics journals, i.e. The Astrophysical Journal, Monthly Notices of the Royal Astronomical Society, Astronomy and Astrophysics, and The Astronomical Journal. These four journals were chosen because, on the one hand, they are the most internationally prestigious journals in Astrophysics and thus have a high impact factor and, on the other hand, they publish RPs on a wide variety of major issues in this discipline. Interestingly, a prelude to the presence of abbreviations in the field of Astrophysics is the fact that the above-mentioned journals are usually known, both in their written and spoken forms, by their abbreviated names: ApJ (The Astrophysical Journal), MNRAS (The Monthly Notices of the Royal Astronomical Society), A\&A (Astronomy and Astrophysics), and AJ (The Astronomical Journal) ${ }^{2}$.

Before starting our analysis, we looked into the guidelines of each journal in order to check the information regarding

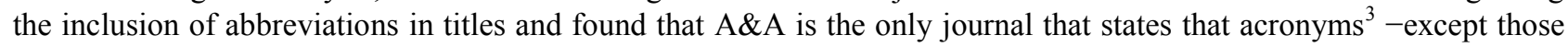
that are in general use- should be avoided. None of the other three journals provides any information related to the use of abbreviations.

In order to conduct our diachronic analysis, we manually and randomly collected all the abbreviations included in the RP titles from three different periods comprising 100 RPs each: Block A (1998), Block B (2004), and Block C (2012). The 100 titles per block comprise 25 RPs per journal and block, i.e. a total of 75 RPs per journal and a grand total of 300 RPs. We then counted capitalized abbreviations according to their semantic components. So, 'LMC' ( $<$ Large Magellanic Cloud) was counted as three words, SCUBA' $(<$ Submillimeter Common-User Bolometer Array) as five words, and 'Cas' (< Casiopea) as one word. Lexicalized acronyms were counted as one word: 'quasar' (< quasi-stellar radio source) was counted as one word. Finally, so as to determine whether the paired two-sample differences observed were statistically significant or not, we analyzed our data by means of the parametric Student's $t$-test. The alpha value was set at 0.05 . 


\section{Results}

\subsection{Quantitative analysis}

Table 1. Total and average number of abbreviations per RP title in the whole sample, per block and journal

\begin{tabular}{ccccc}
\hline Journal & Block A & Block B & Block C & Total \\
\hline AJ & $33(1.32)$ & $67(2.68)$ & $22(0.88)$ & $122(1.63)$ \\
\hline A\&A & $45(1.80)$ & $44(1.76)$ & $22(0.88)$ & $111(1.48)$ \\
\hline MNRAS & $22(0.88)$ & $20(0.80)$ & $31(1.24)$ & $73(0.97)$ \\
\hline ApJ & $13(0.52)$ & $24(0.96)$ & $21(0.84)$ & $58(0.77)$ \\
\hline Total & $113(1.13)$ & $155(1.55)$ & $96(0.96)$ & $364(1.21)$ \\
\hline
\end{tabular}

The 300 titles analysed in this paper consist of 4047 words of which 364 are abbreviations (Table 1), i.e. abbreviations account for $8.99 \%$ of the total title length and the average number of abbreviations per title is 1.21 .

If we examine our results per journal, abbreviations are more frequent in AJ and A\&A, far away from MNRAS and ApJ, to the point that abbreviations in AJ amount more than twice as much as in ApJ. In global diachronic terms, abbreviations increase in Block B and fall in Block $\mathrm{C}$ at a level even lower than that of Block A. The variation pattern of abbreviations is the same in AJ and ApJ (a rise in Block B and a fall in Block C) and runs parallel to the title length behaviour previously noted by Méndez, Alcaraz, and Salager-Meyer (2014a). Conversely, it does not run parallel in A\&A and MNRAS since the number of abbreviations in A\&A steadily decreases from Block A to Block C and in MNRAS it falls in Block B and rises in Block C.

Figure 1 displays the mean value of the number of abbreviations divided by the number of words per RP title for the four journals and the three blocks.

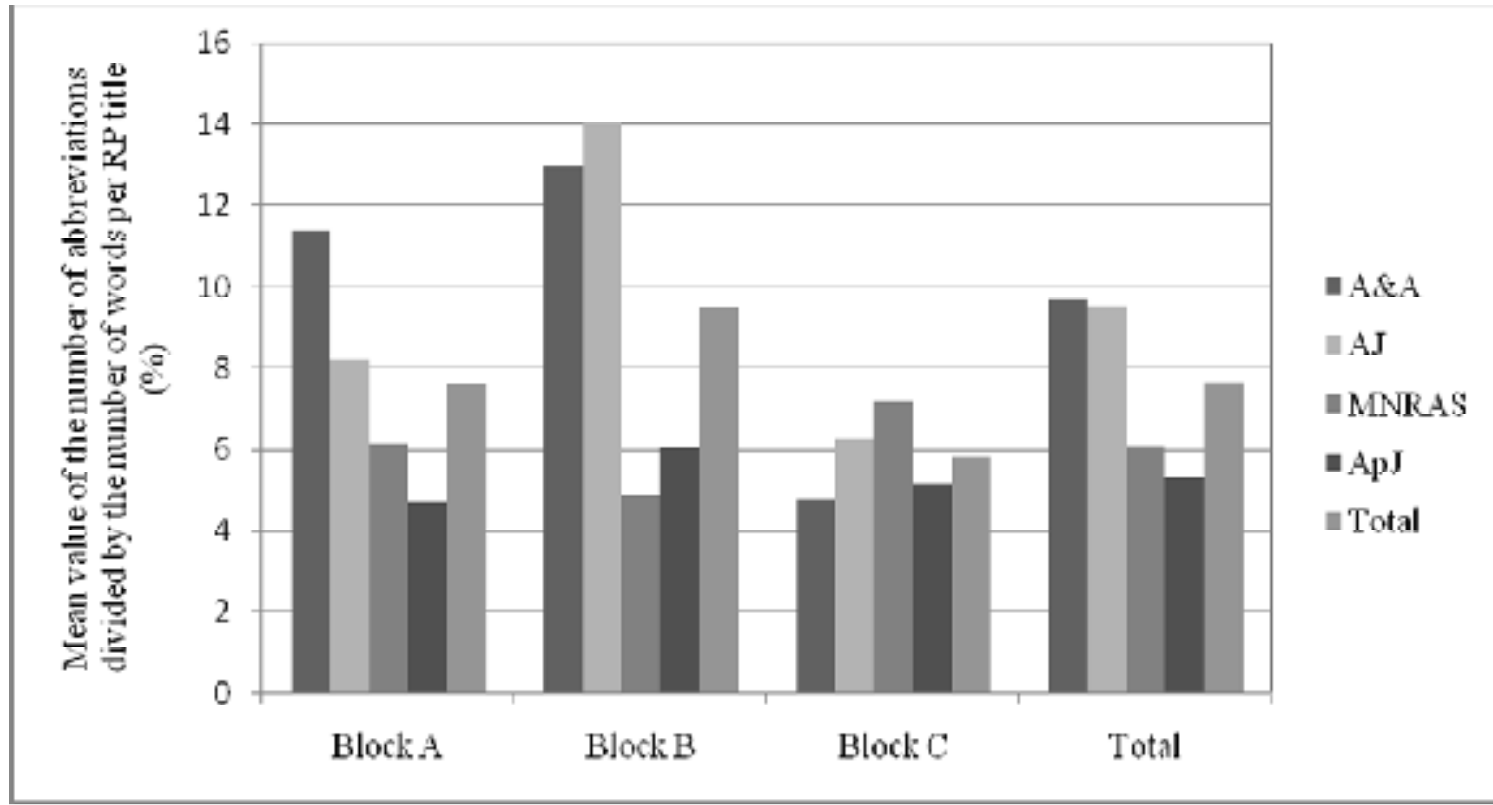

Figure 1. Mean value of the number of abbreviations divided by the number of words per RP title, per block and journal (percentage-wise)

The difference in the mean value of the number of abbreviations/number of words per RP title in the whole corpus between Blocks $\mathrm{B}$ and $\mathrm{C}$ is statistically significant $(9.48 \%$ to $5.85 \% \mathrm{p}=0.015)$. The difference is also statistically significant between Blocks $\mathrm{A}$ and $\mathrm{C}$ in $\mathrm{A} \& \mathrm{~A}(11.38 \%$ to $4.78 \% \mathrm{p}=0.024)$ and between Blocks $\mathrm{B}$ and $\mathrm{C}(14.06 \%$ to $6.27 \%, p=0.012)$ in AJ. Likewise, there is a statistically significant difference in the mean value of the number of abbreviations/number of words per RP title between A\&A and ApJ (9.71\% and 5.30\%, p=0.017).

Since the process of shortening is very productive for word formation, many linguists have devoted their time and effort to study it. Descriptively speaking, abbreviations have usually been categorized into the following types (Bauer, 1983; Cannon, 1989; Algeo, 1993, 2007; Rodríguez \& Cannon, 1994; Fischer, 1998; López Rúa, 2002; Plag, 2003, among many others):

1) Initialisms or alphabetisms, i.e. the simplest abbreviations usually made up of the first letter(s) of content (lexical) words in complex expressions formed by more than one term or of some of the syllables of polysyllabic words; 
2) Acronyms, i.e. shortened forms with a syllabic structure;

3) Blends or combinations of the beginning of one word and the end of another;

4) Clippings, i.e. a variety of shortening which consists in the reduction of a word to one of its parts.

Table 2. Total number of initialisms in the whole sample, per block and journal

\begin{tabular}{ccccc}
\hline Journal & Block A & Block B & Block C & Total \\
\hline AJ & 29 & 59 & 17 & 105 \\
\hline A\&A & 41 & 41 & 18 & 100 \\
\hline MNRAS & 18 & 14 & 27 & 59 \\
\hline ApJ & 11 & 20 & 17 & 48 \\
\hline Total & 99 & 134 & 79 & 312 \\
\hline
\end{tabular}

Table 3. Total number of acronyms in the whole sample, per block and journal

\begin{tabular}{ccccc}
\hline Journal & Block A & Block B & Block C & Total \\
\hline AJ & 4 & 8 & 5 & 17 \\
\hline A\&A & 3 & 2 & 4 & 9 \\
\hline MNRAS & 4 & 5 & 4 & 13 \\
\hline ApJ & 2 & 4 & 4 & 49 \\
\hline Total & 13 & 19 & 17 & 49 \\
\hline
\end{tabular}

Table 4. Total number of clippings in the whole sample, per block and journal

\begin{tabular}{ccccc}
\hline Journal & Block A & Block B & Block C & Total \\
\hline AJ & 0 & 0 & 0 & 0 \\
\hline A\&A & 1 & 1 & 0 & 2 \\
\hline MNRAS & 0 & 1 & 0 & 0 \\
\hline ApJ & 0 & 0 & 0 & 3 \\
\hline Total & 1 & 2 & 0 & 1 \\
\hline
\end{tabular}

Table 2, Table 3, and Table 4 show the number of initialisms, acronyms and clippings that make up the 364 abbreviations found in the 300 titles, per block and journal. As it can be seen, initialisms have $85.71 \%$, acronyms have $13.46 \%$ and clippings have $0.83 \%$ of the share (check Figure 2). No blend was found in our corpus.

Initialisms (Table 2), acronyms (Table 3) and clippings (Table 4) follow the same trend as the one observed in the total number of abbreviations (Table 1), i.e. their number increases in Block B and decreases in Block C. Moreover, initialisms are more frequent in $\mathrm{AJ}$ and $\mathrm{A} \& \mathrm{~A}$, while acronyms are more numerous in $\mathrm{AJ}$ an MNRAS and clippings have been traced only in A\&A (Block A and B) and MNRAS (Block B), not even a single case having been found in Block C.

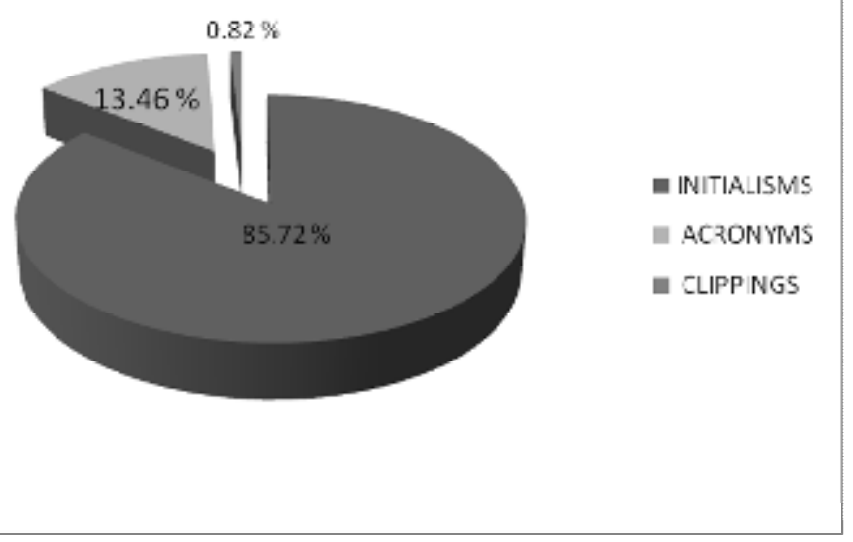

Figure 2. Distribution of abbreviations in the whole sample 


\subsection{Qualitative analysis}

Here-below are some examples of initialisms, acronyms and clippings, as well as the different variations and peculiarities that were found in our sample.

\subsubsection{Initialisms}

Particularly common in scientific fields are initialisms, whose resulting letters are written in upper-case form, are sounded one by one, and usually range from one to five representations. The following title contains a three-letter initialism:

(1) Ionized gas kinematics and massive star formation in NGC $1530\left(A \& A-B^{4}\right)$

The initialism NGC, which stands for "New General Catalogue" (of Nebulae and Clusters of Stars), is characterized by the linguistic phenomenon of 'ellipsis', i.e. the missing out of one or more words from the original expression. In this example, NGC is qualified by the Arabic number 1530 that refers to an object of this catalogue.

The same elliptical phenomenon can be appreciated in the following initialism:

(2) Hunting for young dispersing star clusters in IC 2574 (AJ-C)

IC represents "Index Catalogue" (of Nebulae and Clusters of Stars), applied to a catalogue of galaxies, nebulae and stellar cumuli that supplements the New General Catalogue. Like in NGC, the Arabic number 2574 designs one of the IC objects, which is also known as Coddington's Nebula because it was discovered on 17the April 1898 by the US astronomer Edwin Foster Coddington.

It is interesting to add that both NGC and IC are forms used by the international Astrophysics community at large.

Apart from Arabic numbers, initialisms may also be combined with Roman numerals, as in the case of SDSS $(<$ Sloan Digital Sky Survey):

(3) New neutrino mass bounds from SDSS-III data release and photometric luminous galaxies (AJ-C)

SDSS is an eponymous initialism since it conceals the proper name of Alfred P. Sloan Jr., a US business executive in the automotive industry.

Like general terms, proper names or eponyms ${ }^{5}$ are also abbreviated. The following title is worth commenting upon because it displays some interesting shortening features:

(4) Comprehensive modelling of the planetary nebula LMC-SMP61 and its WC-type central star (A\&A-B)

LMC is the abridged form of 'Large Magellanic Cloud', the eponymous derivative 'Magellanic' being named after the Portuguese explorer Ferdinand Magellan. LMC is combined with another eponymous initialism, SMP, which corresponds to the shortened surnames of the astronomers N. Sanduleak, D. J. McConnell and A. G. Davis Philip. SMP is followed by an Arabic numeral that names one of the objects contained in the catalogue compiled by the three referred scientists. WC, the third initialism included in this title, suffers from one omission as it represents the usual abridged eponymous expression "WR", named after the French astronomers Charles Wolf and Georges Rayet, and 'C' is the symbol of a chemical element (Carbon) ${ }^{6}$.

On some occasions, both WR and LMC are also found in their non-truncated form:

(5) On the physical model of dust around Wolf-Rayet stars (MNRAS-A)

(6) An intriguing X-ray arc surrounding the X-ray source RX J053335-6854.9 toward the large Magellanic cloud (AJB)

Besides the full expanded expression 'large Magellanic cloud', title (6) contains two initialisms: 'R', which corresponds to the abridged form of 'Radio', and ' $\mathrm{J}$ ', which stands for the Julian calendar that was introduced by Julius Caesar in $46 \mathrm{BC}$ as a reform of the Roman calendar. ' $\mathrm{X}$ ' is the symbol applied to an emission of an unknown nature and the Arabic numbers that follow the initialism ' $\mathrm{J}$ ' correspond to coordinates in the sky (right ascension and declination). It is worth pointing out that in expressions consisting of letters and numbers, the latter usually designate sky coordinates.

It may even happen that WR appears both in its abbreviated and spelled out forms in the same title:

(7) WR 121 obscured by a dust cloud: the key to understanding occasional "eclipses" of "dusty" Wolf-Rayet WC stars (A\&A-A)

Some eponymous abbreviations may not follow the usual trend:

(8) A Ly $\alpha$ emitter with an extremely large rest-frame equivalent width of $\sim 900 \AA$ at $\mathrm{z}=6.5$ : a candidate population IIIdominated galaxy? (ApJ-C)

Apart from the mathematical element ' $\sim$ ' and the magnitude ' $z$ ', which is applied to the phenomenon observed when the wavelength of light or of any other electromagnetic radiation from an object is increased or shifted to the red end of the spectrum, this example contains the following initialisms:

- 'Ly', which stands for the shortened eponym of the American physicist and spectroscopist Theodore Lyman, together with the Greek letter ' $\alpha$ ',

- ‘ $\AA$ ', the symbol of 'Angström', a unit of length named after the Swedish physicist Anders Jonas Angström. 
Although initialisms are usually built up with the initials of content words (nouns, verbs, adverbs, and adjectives), on some occasions the initials of some of these constituents may be omitted:

(9) XMM-Newton observations of PSR B1706-44 (ApJ-B)

In XMM (< X-ray Multi-mirror Mission), neither 'ray' nor 'mirror' 'have been included. In the case of 'ray', its exclusion is probably due to the fact that it is a standard term known to all Astrophysicists, whereas 'mirror' has not been included, likely for the sake of shortening the initialism. The initialism PSR is the result of a double truncation: 'pulsating star' > pulsar > PSR (see the here-below sub-sections on Acronyms and Blends). Finally, 'B' stands for 'Besselian equinox' named after the $19^{\text {th }}$ German astronomer and mathematician Friedrich Bessel.

The graphic inconsistency already observed in example (8), in which the initialism contains the two first letters of a single eponym, is particularly noticeable in the following titles:

(10) Prospects for galaxy-mass relations from the 6dF Galaxy Survey (MNRAS-B)

(11) 20 CVn: simultaneous UVBY photometry (A\&A-A)

(12) X-ray flares from Sgr A: Star-disk interactions? (A\&A-B)

'dF' [title (10)], 'CVn' [title (11)], and 'Sgr' [title (12)] are the abridged forms of the expressions 'degree Field', 'Canum VeNaticorum' and 'Sagittarius', respectively. In example (12), the capital letters ' $U$ ', 'V', 'B', and ' $\mathrm{Y}$ ' designate different types of photometric filters, although only 'U' (< Ultraviolet), 'V' ( $<$ Visual) and 'B' ( Blue) are initialisms, ' $\mathrm{Y}$ ' being an arbitrarily chosen letter referring to one of these filters.

As for Sgr, this form is probably used in order to avoid a clash with its homophone SGR, another shortened form that stands for 'Soft Gamma Repeater':

(13) Proper motions and origins of SGR 1806-20 and SGR $1900+14$ (ApJ-C)

'Sagittarius' may also be found in its non-abbreviated form:

(14) A spectral line survey of selected 3 millimeter bands toward Sagittarius B2 (N-LMH) using the National Radio Astronomy Observatory 12 meter radio telescope and the Berkeley-Illinois-Maryland Association array. I. The observational data (ApJ-B)

In this example, 'N' stands for 'north' and 'LMH' for 'Large Molecule Heimat'. This second initialism conceals the German word 'Heimat' meaning 'home'.

Occasionally, more than five-word initialisms, usually coined $a d$ hoc to address a specific issue, may be found:

(15) CFBDSIR2149-0403: a 4-7 Jupiter-mass free-floating planet in the young moving group AB Doradus? (A\&A-C)

The initialism 'CFBDSIR' refers to the full expression 'Canada-France Brown Dwarfs Survey InfraRed' and thus conceals the initial letters of two toponyms (Canada and France).

The most characteristic feature of the process of shortening, i.e. economy, is exemplified in the following title:

(16) A unified picture of breaks and truncations in spiral galaxies from SDSS and $\mathrm{S}^{4} \mathrm{G}$ imaging (MNRAS-C)

'SDSS' stands for 'Sloan Digital Sky Survey' [see title (3)] and ' $\mathrm{S}^{4} \mathrm{G}$ ' for 'Spitzer Survey of Stellar Structure in Galaxies'. It may be said that $S^{4} G$, which conceals the initial letter of the eponym 'Spitzer', after Lyman Spitzer Jr., a world-renowned theoretical astrophysicist, has been achieved out of a creative thinking. In other words, not only economy but also creativity are often used in the process of shortening.

As for their plural forms, initialisms follow the same procedure as lexicalized nouns, i.e. the 's' mark is added to 'AGN' ( $<$ Active Galactic Nucleus):

(17) A complete sample of soft x-ray-selected AGNs. I. The data (AJ-B)

\subsubsection{Acronyms}

When the string of letters resulting from an abbreviation has a syllabic structure, it is usually pronounced as a word rather than letter-by-letter and we have an acronym. Acronyms are less common than initialisms and do not generally include the initials of function words. Like initialisms, the length of acronyms may have varied representations and may be truncated in different ways. They are usually written in upper-case letters unless they are lexicalized, and therefore written in lower-case letters:

(18) Eclipsing binaries in the OGLE variable star catalog. III. Long-period contact systems (AJ-A)

(19) The 172 ks Chandra exposure of the LALA bootes field: X-ray source catalog (AJ-B)

'OGLE' [example (18)] stands for 'Optical Gravitational Lensing Experiment', whereas example (19) conceals the lexicalized abridged eponymous term 'Chandra', applied to an artificial satellite named after the Indian-American astrophysicist Subrahmanyan Chandrasekhar who won the Nobel Prize for Physics together with William A. Fowler in $1983^{7}$. Example (19) also includes the acronym 'LALA' $(<$ Large Area Lyman Alpha survey) in which the content word 'survey' has been left out. Conversely to example (8), only the initial letter of the proper name 'Lyman' has been kept. Apart from these two acronyms, example (19) also contains the symbol 'ks' ( $<$ KiloSeconds), referring to a unit of time meaning 1000 seconds which, from a linguistic standpoint, has been completely lexicalized since it is written in lowercase letters like any other common word. 
Structure inconsistencies are also observed in acronyms:

(20) Observations from SDO, hinode, and STEREO of a twisting and writhing start to a solar-filament-eruption cascade (AJ-C)

(21) Parsec-scale radio structure of the double active nucleus of NGC 6240 (AJ-B)

Apart from the initialism 'SDO' (< Solar Dynamics Observatory), title (20) contains the Japanese word hinode 'sunrise' and the eye-catching acronym 'STEREO' (< Solar TErrestrial RElations Observatory). As for title (21), the lexicalized acronym 'parsec' ( $<$ PARallax of one arc SECond), which is applied to unit of length in Astronomy, omits not only the initials of the preposition 'of', but also of the adjective 'one' and of the noun 'arc'. 'Parsec' can also be qualified as a 'back clipped acronym' (see following sub-section on clippings) since it consists of the two first halves of a compound expression ${ }^{8}$. 'Parsec' may also be combined with the Greek prefix 'kilo' as in the following title:

(22) The biconical kiloparsec structure generated by nuclear starbursts (MNRAS-A)

The omission of the initial of a content word can also be appreciated in the following acronym:

(23) Millimeter imaging of submillimeter galaxies in the COSMOS field: redshift distribution (A\&A-C)

'COSMOS' may stand for two different spelled out expressions with no difference in meaning, 'COSMic evOlution Survey' and 'COSMOlogical evolution Survey'. It cannot be denied that the acronym perfectly fits within the terminology it belongs to since its homophone 'cosmos' means 'the world or universe considered as an ordered system' and that its authors have shown great creativity when coining it.

Other irregular constructions can be found in the following acronyms:

(24) Observations of binary stars with the differential speckle survey instrument. IV. Observation of Kepler, CoRot, and Hipparcos stars from the Gemini North telescope (AJ-C)

(25) Disappearance of hard X-ray emission in the last BeppoSAX observation of the z source GX 349+2 (ApJ-B)

(26) ROSAT PSPC X-ray observations of the nearby spiral galaxy M83 (A\&A-A)

(27) XMM-Newton observations of two high-redshift quasars: RX J1028-0844 and BR 0351-10341 (AJ-B)

In Example (24), 'CoRoT' (<COnvection ROtation and planetary Transits) is applied to a space mission led by several international partners, whereas 'Hipparcos' $(<$ HIgh Precision PARallax COllecting Satellite) may be qualified as a proper name since the upper-case initial letter has been kept. The use of the first capital letter and the whole acronym itself may also reflect an attempt to make reference to the Greek astronomer, geographer and mathematician Hipparchus of Nicaea. Moreover, the term 'parallax' may be considered an example of 'back clipping' (see the corresponding subsection here-below).

'BeppoSAX' in example (25) is a combination of 'Beppo', the nickname of the Italian physicist Giuseppe "Beppo" Ochialini, and the Italian initialized expression 'SAX' (< Satellite per Astronomia X 'X-Ray Astronomy Satellite').

The complex expression 'ROSAT PSPC' in example (26) contains the acronym 'ROSAT', a mixture of the abridged and anglicized eponym 'RO' ( $<$ Wilhelm RÖntgen, the surname of a German researcher), the clipping 'SAT' ( $<$ SATellite), and the initialism 'PSPC' ( $<$ Positive Sensitive Proportional Counters). Another eponymous initialism contained in this title is 'M83', named after Charles Messier, a French astronomer who published an astronomical catalogue consisting of nebulae and star clusters that came to be known as 'Messier objects'.

As for the lexicalized acronym 'quasar' [< 'QUASi-stellAR (radio source)] in example (27), the content words 'radio' and 'source' have been excluded. 'Quasar', also referred to as 'QSO', may be qualified as a "blend" since it is nearly formed by the beginning of one word and the ending of another one. The first ' $\mathrm{R}$ ' stands for 'Radio' while ' $\mathrm{J}$ ' refers to the Julian calendar [(see title (6)]. 'B' stands for 'Blue' and the second 'R' for 'Red', which are applied to two different types of photometric filters.

Like in the case of the doubly truncated 'pc', the acronym 'ROSAT' has been again abridged to form new truncated forms:

(28) Temperature gradients in XMM-Newton observed REFLEX-DXL galaxy clusters at $\mathrm{z} \sim 0.3$ (A\&A-B)

(29) Chandra and XMM-Newton observations of RDCS 1252.9-2927, a massive cluster at $\mathrm{z}=1.241$ (AJ-B)

In title (28), the creative and appealing 'REFLEX' is constituted by ' $R$ ' $[<\mathrm{R}(\mathrm{OSAT})]$, 'E' $[<\mathrm{E}(\mathrm{SO})<\mathrm{European}$ Southern Observatory], 'F' (< Flux), 'L' (< Limited), 'E', which has been added to make the word pronounceable, and ' $\mathrm{X}$ ' (ray)'. 'REFLEX' is moreover combined with the initialism 'DXL' that stands for the expression 'Distant X-(ray) Luminous'. In both cases, the content word 'ray' has been omitted [see title (9)].

As for the initialism 'RDCS' in title (29), it corresponds to 'R(OSAT) Deep Cluster Survey'.

Like in example (15), some toponyms are found in acronymic shortenings:

(30) Synthetic observations of first hydrostatic cores in collapsing low-mass dense cores II. Simulated ALMA dust emission maps (A\&A-C)

(31) Aqueye optical observations of the Crab Nebular pulsar (A\&A-C)

'ALMA' stands for 'Atacama Large Millimeter Array', an astronomical interferometer of radio telescopes in $\underline{\text { Atacama }}$ desert in northern Chile (South America), whereas 'Aqueye' ( $<$ Asiago Quantum eye) combines the initial letter of the 
Italian town of Asiago (this is why it is written with an initial capital letter), the two first letters of the Latin word 'quantum' and the full English word 'eye'.

The plural mark of acronyms follows the same rule as lexicalized words (and initialisms), i.e. the 's' ending is added to the shortened forms:

(32) Probing the mass fraction of MACHOs in extragalactic halos (ApJ-A)

It may be argued that 'MACHOs', which stands for 'MAssive Compact Halo Objects', has been coined for the sake of appealing to the reader since in everyday language this Spanish colloquial word borrowed by English denotes pride in characteristics believed to be typically masculine (physical strength, sexual appetite, etc.).

Other acronyms built up in a creative and witty manner are found in the following titles:

(33) Observations of the diffuse near-IR sky emission with a balloon-borne infrared Telescope (TRIP) (A\&A-A)

(34) A new analysis of theWASP-3 system: no evidence for an additional companion (MNRAS-C)

In example (33), the meaning of the acronym 'TRIP' is explained in the title, although the usual pattern and order for word shortening have not been followed since the acronym has been made up with the following elements: ' $\mathrm{T}$ ' $(<$ Telescope), 'R' ( $<$ 'red'), 'I' (< Infra), and 'P', which is non-existent in the original non-truncated expression. It may be speculated that the coiners of this acronym probably included the ' $\mathrm{P}$ ' letter as they wanted to refer to an outward and return journey and at the same time to introduce a sort of word game by hinting at a hallucinogenic experience in the jargon of drugs, which is colloquially known as 'trip'.

As for the acronym 'WASP' ( $<$ Wide Angle Search for Planets) in example (34), the creation of playfulness may also be reached by its spelling similarity with 'WASP' that may stand for 'White, Anglo-Saxon and Protestant', 'Web Applications and Service Providers', 'Web Analytics Solution Profiler', or by its homophone similarity with the common word 'wasp'.

Another acronym that clearly does not follow the general trend of excluding the initial letters of function words is 'ANDICAM' (< A Novel Double Imaging CAMera), which contains the indefinite determiner "a", probably with the purpose to make the acronym more manageable, and the clipped word 'CAM' (see corresponding sub-section below):

(35) Optical-infrared ANDICAM observations of the transient associated with GRB 030329 (AJ-B)

This title also includes the initialism 'GRB' ( $<$ Gamma Ray Burst).

\subsubsection{Clippings}

Since 'Par' [ $<$ Parallax, title (24)], SAT [ $<$ SATellite, title (26)], and 'CAM' [< CAMera, title (35)] have already been assimilated in the acronyms 'Hipparcos', 'ROSAT', and 'ANDICAM, respectively', it can be said that only two clipped forms ('Cas' and 'Oph') have been traced in the following titles:

(37) The eclipsing binary star RZ Cas

I. First spectroscopic detection of rapid pulsations in an Algol system? (A\&A-B)

(38) Long-term photometric study of the WUMa binary star V523 Cas (MNRAS-B)

(39) Spectroscopic and spectrophotometric studies of V2301 Oph (A\&A-A)

'Cas' (< Casiopea) and 'Oph' (< Ophiuchus) can be qualified as 'back clippings' because only their first syllable has been kept. Both are also proper names as they are written with an initial capital letter. No examples of fore or middle clippings, i.e. clippings that retain the final or middle parts of words, have been found.

\section{Discussion}

Our qualitative analysis of abbreviations has put forward the inclusion of initialisms, acronyms and clippings in RP titles published in the main English-written Astrophysics journals. The variations in spellings and inconsistencies when constructing shortened forms, which were already mentioned in previous studies (Alcaraz, 2001; Biber \& Clark, 2002; Biber, 2003; Leech, Hundt, Mair, \& Smith, 2009, to name just a few), have also been noticed: mixtures of lower and upper-case letters, omissions of content words and presence of function words, initialisms and fully lexicalized acronyms that conceal the initial letters of eponyms and toponyms, conversion of some of them into internationalisms, same plural forms as those of common words, and combination of words from different origins.

From a functional role of language (Halliday, 1994), it may be said that the main reasons behind abbreviations as a short-hand when writing are to achieve linguistic economy by avoiding writing out the full word/group of words, to highlight field specialty, and to give their users a separate identity (Kasesniemi \& Rautianen, 2002; Ling \& Yttri, 2002; Crystal, 2003; Thurlow \& Brown, 2003). In RP titles, the main aim of which is to include an information load in the most concise and condensed form (Haggan, 2004; Wang \& Bai, 2007; Soler, 2007, among others), we may argue that, at least in Astrophysics, saving space and time goes hand in hand with a sort of creativity and wittiness in order to arouse readers' curiosity and push them to read the whole paper.

In spite of the convenience, creativity and innovation displayed in the coinage of many abbreviations, some dangers are nevertheless on the watch of their use. One of them is the presence of some abbreviations in force within certain specific groups but not adopted by the whole Astrophysics community. It has often been stated that for the first reference of an abbreviation in a text, the term to which it stands for should be used in full with the abbreviation included in brackets, and it should be checked that the new created form has not been previously applied to another 
concept, at least in the same field. Because of their own characteristics (maximum conciseness and precision), titles do not allow the full expansion of the abbreviations they refer to, and no doubt that it is easier and more economical both from a written and spoken standpoint to write and say 'NGC' than 'New General Catalogue' (of Nebulae and Clusters of Stars) or 'ROSAT PSPC' than 'Röntgen Satellite Positive Sensitive Proportional Counters'.

Another danger refers to the ambiguity of some abbreviations that may lead to misinterpretations: does the nonlexicalized acronym 'WASP' stand for 'Wide Angle Search for Planets' or for 'Wideband Analog Spectrometer'? Do the homophones 'Sgr' and 'SGR' refer to 'Sagittarius' or 'Soft Gamma Repeater'? Or does the initialism 'SMP' correspond to 'Sanduleak, McConnell, Philip' or 'Selected Minor Planet'? This is why the rules stated by A\&A should be applied when including abbreviations in Astrophysics titles ${ }^{9}$, i.e. titles should only include standard abbreviations whose meaning is known to the whole Astrophysics community such as, for example, 'Hipparcos', 'NGC', or 'parsec', to name just a few ${ }^{10}$.

It is also important to mention that the use of expanded or shortened forms may depend on the authors' intention. For instance, we could speculate that authors tend to use non-abbreviated forms when they want to refer to more generic items or when they want to emphasize a concept with respect to another one. Likewise, the (non)-use of abbreviations may be related to authors' expertise within a specific branch of Astrophysics (solar, planetary, stellar, galactic, etc.) or may even reflect their individual stylistic preferences. However, to be proved or refuted, all these hypotheses would have to be based on a more comprehensive study of a larger corpus of abbreviations, including personal interviews. All in all, we think that there is a clear need for the establishment of a standard procedure to avoid confusion when using abbreviations in titles and to improve clarity among novice and/or expert readers and writers. This is why the creation of a commission of experts both in linguistic and astrophysical matters would be recommended. Linguists and astrophysicists should work hand by hand to develop proper abbreviation rules. Once developed, these rules should be spread within the astrophysical community. Journal editors would also have their role to play in limiting the use of abbreviations in titles to the only well-known ones.

In addition, their almost omnipresent occurrence in Astrophysics should deserve their gathering in a comprehensive open database that the Astrophysics community and any other scientific community may access free online. A similar initiative was already put forward by the International Astronomical Union (IAU) when in their 2012 assembly they requested the creation of a list of astronomical "acronyms" on the Wikipedia. Nevertheless, nothing was mentioned about the linguistic aspects associated to the creation and handling of abbreviations. In this regard, the application of the previously developed standard abbreviation rules before incorporating new abbreviated forms to the proposed open database would definitely improve their understanding and avoid any problems that may arise from their use. Finally, the design of academic courses related to the teaching of abbreviations within the field of Astrophysics would be advisable so as to help novice and/or expert readers and writers. Altogether these options would lead to a better interaction among the different branches of Astrophysics in particular and of science in general and would definitely improve how research is performed and communicated.

As for our quantitative analysis, it has revealed that abbreviations abound in Astrophysics titles and that initialisms predominate over acronyms and clippings, which would be in line with Crystal's findings (2003). This should come as no surprise since initialisms are the easier and first step performed when shortening words and groups of words. The coinage of acronyms would need more effort and creativity, while clippings are primarily found in spoken language.

Our findings have also shown that there are more abbreviations in AJ than in the other journals. This result is probably due to the fact that observational matters, i.e. the most experimental part of Astrophysics that require complex instrumentation (telescopes, detection devices, computer hardware and software, or space missions), are more relevant in this journal than in the remaining ones. Furthermore, the use of last generation technology implies the achievement of new observational data of formerly known astronomical objects together with the discovery of new ones whose names would normally appear in abbreviated form. Our quantitative analysis has also shown that the total number of abbreviations increases in Block B and falls in Block C. This fall, mainly led by the US-based AJ, may be accounted for, in our opinion, to the worldwide economic crisis that started in the USA in 2006 (Tully, 2006). The substantial funding allocated to the development of astronomical technology, which had been steadily increasing till then, was reduced as a consequence of that crisis to the point that less money was devoted to research. This meant that smaller projects were launched and that fewer scientific devices, whose denomination is usually shortened in titles, were created.

Another economic crisis has also to be mentioned, and it is the one that started at the beginning of the year 2000, mainly in Germany. After German reunification, the country had to face an excessive deficit and huge economic problems to the point that it was known as "the sick man of Europe" until the year 2005, when its economy began to recover (Dustmann, Fitzenberger, Schönberg, \& Spitz-Oener, 2014). However, it seems that the recovery did not benefit German Astrophysics research in particular and European Astrophysics research in general since many projects were carried out in collaboration with the USA, and this consequence is reflected in the steady decrease noticed in the number of abbreviations in the European-based A\&A.

Due to their more general trend, i.e. not so observationally-oriented, MNRAS and ApJ may have not suffered both economic crises in the same dimension as AJ and A\&A. This fact together with other extra-linguistic factors such as the increase in authorship and collaboration practices over time (Méndez, Alcaraz, \& Salager-Meyer, 2014b; Méndez \& Alcaraz, 2015) may explain why the number of abbreviations is higher in Block C than in Block A for the first two journals. 
Nevertheless, as in the case of our qualitative results, a more detailed analysis with a larger corpus of abbreviations in Astrophysics RP titles would be needed to corroborate all the ideas presented above and would likely provide a more comprehensive understanding of the issue.

\section{Conclusions}

The main results obtained in our study have highlighted that the process of shortening words and groups of words is one of the most characteristic and recurrent features in Astrophysics RP titling construction. In spite of the convenience, creativity and innovation displayed in the coinage of many abbreviations, some of them may pose certain difficulties of understanding and/or misinterpretation because of their specificity, ambiguity, or overlapping. This is why their proper use should be regulated by teams made up of experts in different specialized astrophysical fields, linguists and journal editors. Since Astrophysics is a multidisciplinary subject, there is no doubt that the abbreviation rules established by these experts would quickly reach the whole scientific community and would surely be of great importance in both research and educational spheres.

As for our qualitative analysis, it has disclosed that Astrophysics RP titles include the usual types of abbreviations, i.e. initialisms, acronyms and clippings, which are formed by means of different building patterns: combination of lower and upper-case letters, occasional exclusion of content words and inclusion of function words, presence of chemical and mathematical symbols, mixture of words from different origins, lexicalization of acronyms concealing the initial letters of eponyms and toponyms, international character of some initialisms and acronyms, and formation of plural forms in the same way as common words.

Finally, our quantitative results have showed that initialisms by far outnumber the two other kinds of abbreviations. They have also disclosed that in terms of global evolution, and for all the journals studied, the mean number of abbreviations per RP title runs parallel to the average title length observed in a previous study on the same corpus (see Méndez, Alcaraz, \& Salager-Meyer, 2014a). However and from a specific diachronic perspective, each journal has its own title shortening variation patterns, which we have related to its specific scope and to the socio-economic and political situation in both the European and US geographic contexts.

\section{References}

Alcaraz, M. Á. (2001). Exploring English abbreviations in Medical Spanish. Indian Journal of Applied Linguistics: an International Journal of Applied Language Sciences, 27(1), 43-56.

Algeo, J. (Ed.) (1993). Fifty Years among the New Words: A Dictionary of Neologisms. 1941-1991. Cambridge: Cambridge University Press.

Algeo, J. (2007). Vocabulary. In S. Romaine (Ed.). The Cambridge History of the English Language, Volume IV (17761997) (pp. 57-91). Cambridge: Cambridge University Press. doi: 10.1017/CHOL9780521264778

Anthony, L. (2001). Characteristic features of research article titles in computer science. IEEE Transactions of Professional Communication, 44(3), 187-194. doi: 10.1109/47.946464

Bauer, L. (1983). English Word-Formation. Cambridge: Cambridge University Press. doi:10.1017/CB09781139165846

Biber, D., \& Clark, V. (2002). Historical shifts in modification patterns with complex noun phrase structures: How long can you go without a verb? In T. Fanego, M. López-Couso, \& J. Pérez-Guerra (Eds.). English Historical Syntax and Morphology (pp. 46-53). Amsterdam: John Benjamins. doi: 10.1075/cilt.223.06bib

Biber, D. (2003). Compressed noun phrase structures in newspaper discourse: The competing demands of popularization vs. economy. In J. Aitchison, \& D. Lewis (Eds.). New Media Discourse (pp. 169-181). London: Routledge.

Cannon, G. (1989). Abbreviations and acronyms in English word-formation. American Speech, 64(2), 99- 127. doi: $10.2307 / 455038$

Cheng, S. W., Kuo, C. W., \& Kuo, C. H. (2012). Research article titles in applied linguistics. Journal of Academic Language and Learning, 6(1), A1-A14.

Crystal, D. (2003). The Cambridge Encyclopedia of the English Language. Cambridge: Cambridge University Press.

Dustmann C., Fitzenberger B., Schönberg U., \& Spitz-Oener A. (2014). From sick man of Europe to economic superstar: Germany's resurgent economy. Journal of Economic Perspectives, 28 (1), 167-188. doi:10.1257/jep.28.1.167

Fischer, R. (1998). Lexical Change in Present-Day English. A Corpus-Based Study of the Motivation, Institutionalization, and Productivity of Creative Neologisms (pp. 21-43). Tübingen: Gunter Narr.

Fortanet, I., Coll, J. F., Palmer, J. C., \& Posteguillo, S. (1997). The Writing of titles in academic research articles. In R. M. Chamorro, \& A. R. Navarrete (Eds.), Lenguas Aplicadas a las Ciencias y la Tecnología. Aproximaciones (pp. 155-158). Cáceres (Spain): Universidad de Extremadura, Servicio de Publicaciones.

Fortanet, I., Posteguillo, S., Coll, J. F., \& Palmer, J. C. (1998). Linguistic analysis of research article titles: Disciplinary variations. In I. Vázquez, \& I. Camilleu (Eds.), Perspectivas Pragmáticas en Lingüística Aplicada (pp. 443-447). Zaragoza (Spain): Anubar. 
Haggan, M. (2004). Research paper titles in literature, linguistics and science: Dimensions of attractions. Journal of Pragmatics, 36, 293-317. doi:10.1016/S0378-2166(03)00090-0

Halliday, M. A. K. (1994). Introduction to Functional Grammar. London: Arnold.

Hyland, K. (1998). Hedging in Scientific Research Articles. Amsterdam: Benjamins. doi: 10.1075/pbns.54

Hyland, K. (2000). Disciplinary Discourses. Social Interactions in Academic Writing. London: Longman.

International Astronomical Union (IAU). Astronomical acronyms. http://en.wikipedia.org/wiki/Astronomical_acronyms Jaime-Sisó, M. (2009). Titles or headlines? Anticipating conclusions in biomedical research article titles as a persuasive journalistic strategy to attract busy readers. Miscelánea. A Journal of English and American Studies, 39, $29-54$.

Kasesniemi, E. L., \& Rautiainen, P. (2002). Mobile culture of children and teenagers in Finland. In J. Katz, \& M. Aakhus (Eds.), Perpetual Contact: Mobile Communication, Private Talk, Public Performance (pp. 170-192). Cambridge: Cambridge University Press. doi: 10.1017/CB09780511489471.014

Leech, G., Hundt, M., Mair, C., \& Smith, N. (2009). Change in Contemporary English: a Grammatical Study. Cambridge: Cambridge University Press. doi: 10.1017/CBO9780511642210

Leventhal. P. (2011). Introducing the manuscript writer's column. The Write Stuff, 20(3), 199.

Ling, R., \& Yttri, B. (2002). Hyper-coordination via mobile phones in Norway. In J. Katz, \& M. Aakhus (Eds.), Perceptual Contact. Mobile Communication, Private Talk, Public Performance (pp. 139-169). Cambridge: Cambridge University Press. doi: 10.1017/CB09780511489471.013

López Rúa, P. (2002). On the structure of acronyms and neighbouring categories: A prototype-based account. English Language and Linguistics, 6, 31-60. doi: 10.1017/S136067430200103X

Méndez, D. I., Alcaraz, M. Á., \& Salager-Meyer, F. (2014a). Titles in English-medium Astrophysics research articles. Scientometrics, 98, 2331-2351. doi: 10.1007/s11192-013-1174-6

Méndez, D. I., Alcaraz, M. Á., \& Salager-Meyer, F. (2014b). Evaluating authorship variation in English-medium Astrophysics research papers: An across journal and diachronic study (1998-2012). Revista Canaria de Estudios Ingleses, 69, 51-63.

Méndez, D. I., \& Alcaraz, M. Á. (2015). Simultaneous and inter-comparable numerical indicators of collaboration practices in English-medium Astrophysics research papers. Submitted to Information Research.

Peat, J., Elliot, E., Baur, L., \& Keena, V. (2002). Scientific Writing: Easy when you Know How. London: BMJ Books.

Pedersen, O. (2009). Early Physics and Astronomy: A Historical Introduction (4th ed.) Cambridge: Cambridge University Press.

Plag, I. (2003). Word-Formation in English. Cambridge: Cambridge University Press. doi: $10.1017 /$ CBO9780511841323

Publishing Research Consortium (2011). European Science Editing, 37(1), 21. Retrieved from http://www.ease.org.uk/sites/default/files/february_2011_371.pdf

Rodríguez, F., \& Cannon, G. (1994). Remarks on the origin and evolution of abbreviations and acronyms. In F. Fernández, H. M. Fuster, \& J. J. Calvo (Eds.), English Historical Linguistics (pp. 261-272). Amsterdam: Benjamins.

Science Daily. (2008). Scientific Journals Ranked by Field Based on Quality. Retrieved from http://www.science daily.com/releases/2008/02/080227092729.htm.

Soler, V. (2007). Writing titles in science: An exploratory study. English for Specific Purposes, 26(1), 90-102. doi: 10.1016/j.esp.2006.08.001

Soler, V. (2011). Comparative and contrastive observations on scientific titles in written English and Spanish. English for Specific Purposes, 30(2), 124-137. 2): 124-137. doi: 10.1016/j.esp.2010.09.002

Swales, J. M. (1990). Genre Analysis: English in Academic and Research Settings. Cambridge: Cambridge University Press.

Tarone, E., Dwyer, S., Gillette, S., \& Icke, V. (1998). On the use of passive in two Astrophysics journal papers: With Extensions to Other Languages and Other Fields. English for Specific Purposes, 17(1), 113-132. doi: 10.1016/S08894906(97)00032-X

Thurlow, C., \& Brown, A. (2003). Generation Txt? Exposing the sociolinguistics of young people's text- messaging. Retrieved from http://faculty.washington.edu/thurlow/papers/Thurlow(2003)-DAOL.pdf

Tully, S. (2006). Welcome to the dead zone. Fortune. Retrieved from http://money.cnn.com/2006/05/03/news/economy/realstateguide_fortune/

Wang, Y., \& and Bai, Y. (2007). A corpus-based syntactic study of medical research article titles. System, 35, 388-399. doi:10.1016/j.system.2007.01.005 
Notes:

${ }^{1}$ For an excellent review of the literature on the subject see Jaime-Sisó (2009) and Soler (2011).

${ }^{2}$ The Impact Factors listed in the journal home page refer to the year 2012 (A\&A 5.084, AJ 4.965, ApJ 6.733, and MNRAS 5.521).

${ }^{3}$ In the case of A\&A, the term "acronym" presumably applies to all types of abbreviations, without any distinction between them.

4 'A\&A' refers to the journal and 'B' to the block (the year 2004) which the example was drawn from.

${ }^{5}$ An eponym is the name of a person after which something is named, whereas a toponym is the name of a place after which something is named.

${ }^{6}$ Since astronomy is tightly related to other branches of knowledge, it is not surprising that its terminology includes a large number of terms proper of mathematics, chemistry, geology, meteorology, or mythology, among others.

7 The Chandra satellite was formerly known as 'AXAF' ( $<$ Advanced X-ray Astronomical Facility) and the word 'Chandra' also means "moon" in Sanscrit language.

${ }^{8}$ Another form of designating the internationalism 'parsec' is the symbol 'pc' in which the already abridged form has suffered from a contraction by dropping the middle letters and keeping only the first and last ones.

${ }^{9}$ In other scientific fields such as that of medicine, where abbreviations are very common, it has been suggested that abbreviations should be avoided either in abstracts or in titles (Peat et al., 2002).

${ }^{10}$ Many Astrophysics abbreviations can be accessed by non-specialists on online links such as, for example, Acronyms \& Abbreviations (http://www.abbreviations.com/acronyms/ASTRO), Frequently seen space/acronyms (tla.surly.org), or Glossary of terms and Astronomy acronyms (www.iceinspace.com.au/glossary.html). 